\title{
Development of a clinical scoring system for bovine respiratory disease in weaned dairy calves
}

\author{
Gabriele U. Maier, ${ }^{1}$ Joan D. Rowe, ${ }^{2}$ Terry W. Lehenbauer, ${ }^{1,2}$ Betsy M. Karle, ${ }^{3}$ Deniece R. Williams, ${ }^{1}$ \\ John D. Champagne, ${ }^{1}$ and Sharif S. Aly ${ }^{1,2 *}$ \\ ${ }^{1}$ Veterinary Medicine Teaching and Research Center, School of Veterinary Medicine, University of California, Davis, Tulare 93274 \\ ${ }^{2}$ Department of Population Health and Reproduction, School of Veterinary Medicine, University of California, Davis 95616 \\ ${ }^{3}$ Cooperative Extension, Division of Agriculture and Natural Resources, University of California, Orland 95963
}

\section{ABSTRACT}

Clinical scoring systems for bovine respiratory disease (BRD) in weaned dairy calves have been developed in the past with calves experimentally infected with specific respiratory pathogens. In this prevalent case control study, a BRD clinical scoring system for weaned calves was developed using field data from 689 dairy calves housed in group pens on 5 dairies in California. Of the 689 calves in the study, 89 were selected because they appeared sick based on the display of lethargy, depression, or separation from the group, whereas the remaining 600 were randomly selected. Clinical signs were recorded for all calves, and BRD case status was determined by thoracic auscultation and ultrasound examinations, which were interpreted in parallel. Of the 689 calves, 238 were identified as BRD cases. Five survey-adjusted generalized linear mixed models with a logit link function, calf as the unit of analysis, and dairy as a random intercept were assessed using 3-fold cross-validation. The best model chosen based on performance and parsimony contained the variables cough (2 points), abnormal respiration (1 point), low body condition (5 points), sunken eyes (4 points), and a 24 -h ambient temperature range $>15^{\circ} \mathrm{C}$ (1 point) with a 2 -point cutoff for a BRD suspect score. An alternative model did not contain a score for the covariate 24-h ambient temperature range and had a 1-point cutoff. The best model was tested on 174 observations not used for model training and resulted in $77.0 \%$ screening sensitivity, $100 \%$ diagnostic sensitivity, and $61.9 \%$ specificity. Adding rectal temperature $\geq 39.2^{\circ} \mathrm{C}\left(102.5^{\circ} \mathrm{F}\right)$ as a second-tier test increased specificity to $76.7 \%$ and lowered the screening sensitivity to $64.8 \%$ and diagnostic sensitivity to $76.9 \%$. The alternative model had a screening sensitivity of $84.2 \%$, diagnostic sensitivity of

Received July 31, 2018.

Accepted November 19, 2018

*Corresponding author: saly@ucdavis.edu
$100 \%$, and specificity of $45.7 \%$. Adding rectal temperature $\geq 39.2^{\circ} \mathrm{C}\left(102.5^{\circ} \mathrm{F}\right)$ as a second-tier test for scorepositive animals improved specificity of the alternative model to $62.6 \%$ while lowering its screening sensitivity to $70.5 \%$ and diagnostic sensitivity to $76.9 \%$. Use of a 2-tier California BRD postweaning scoring system may provide producers and veterinarians with a new tool to monitor BRD in group-housed dairy calves. Furthermore, the scoring system may aid in judicious medical intervention for BRD cases and reduce unnecessary treatments of animals with antimicrobials.

Key words: dairy calf, bovine respiratory disease, clinical scoring system, bovine respiratory disease

\section{INTRODUCTION}

Bovine respiratory disease (BRD) continues to affect the welfare of dairy calves and the economics of dairies (Panciera and Confer, 2010). As a common cause of morbidity and mortality in dairy calves, BRD was responsible for $46.5 \%$ of calf deaths after weaning (USDA, 2010) and was observed in $11.2 \%$ of weaned calves on heifer-raising operations (USDA, 2012). The negative effects of BRD including financial losses reach beyond the treatment costs of clinically ill animals and include reduced growth, reduced milk production, impaired fertility, and premature culling in the first lactation (McGuirk and Peek, 2014).

Despite the availability of numerous vaccines and antimicrobials for the treatment of the disease, observed incidence rates for BRD in dairy calves have practically remained unchanged over the past few decades (Gorden and Plummer, 2010). Moreover, more than 50\% of pulmonary lesions found at slaughter in feedlot steers were in calves that were never treated for respiratory disease (Wittum et al., 1996; Gardner et al., 1999). The prompt and correct diagnosis of BRD is challenging on farms, especially in weaned dairy calves. Weaned dairy calves are typically housed in larger group pens where there are often inadequate restraint facilities and 
where it is more difficult to monitor them closely. As a result of the challenges in diagnosing BRD on the farm, cases may become neglected until they are more severe. Most on-farm BRD treatment decisions in calves are based on subjective evaluations of respiratory signs and are competing with additional on-farm duties. Furthermore, the criteria for evaluation of BRD vary widely and lead to delayed or missed detection in sick animals as well as to unnecessary treatment of animals with antimicrobial drugs (Abutarbush et al., 2012).

The reference standard for diagnosing BRD is necropsy in combination with histopathological, microbiological, molecular, and biochemical testing methods. In the live animal, thoracic ultrasound and radiography can be used to diagnose BRD but require expensive equipment and operator training (Masseau et al., 2008; Abutarbush et al., 2012). Various other diagnostic tests, such as nasal swabs, transtracheal wash, or bronchoalveolar lavage, provide delayed diagnosis and are technically difficult to perform or prohibitively expensive to be employed on a daily basis on farms (Doyle et al., 2017).

Clinical scoring systems provide an objective way for farm personnel to make treatment decisions that do not require expensive equipment or diagnostics. Several clinical scoring systems to detect BRD in calves have been published. The earliest published BRD detection system by Thomas et al. (1977) relied on hematologic data among other predictors and was used in calves experimentally inoculated with bovine respiratory syncytial virus (BRSV) or bovine viral diarrhea virus. Another early system that was specifically developed for the detection of calves infected with BRSV relied on auscultation findings and presence of abdominal breathing (Verhoeff et al., 1985). A system developed by assessing pulmonary function of calves with chronic respiratory disease and comparing them to predicted values (Collie, 1992) was adapted to score calves experimentally infected with BRSV (Gershwin et al., 2011) and assesses anorexia, depression, cough, nasal discharge, adenitis, conjunctivitis, ocular exudate, rectal temperature, dyspnea, mouth breathing, and auscultation findings. A system adapted from feedlot practice to detect clinical disease in weaned Holstein calves experimentally infected with Mycoplasma bovis used a severity scale from 1 to 4 based on signs of depression, cough, and labored breathing (Amrine et al., 2013). A system developed at the University of Wisconsin for use in young dairy calves used a grading system of 0 to 3 for the clinical signs rectal temperature, nasal discharge, cough, eye discharge, and ear position (McGuirk and Peek, 2014). Finally, in a system for preweaned calves from the University of California, ocular discharge, nasal discharge, ear droop or head tilt, cough, breathing quality, and rectal temperature are evaluated and a weighted score assigned (Love et al., 2014, 2016).

The objective of the present study was to develop and validate a BRD scoring system specifically for weaned dairy calves housed in group pens in an onfarm setting that does not require handling of calves for preliminary diagnosis. The hypothesis was that clinical signs in postweaned dairy calves would be correlated with a premortem diagnosis of BRD.

\section{MATERIALS AND METHODS}

\section{Study Population}

Study calves were enrolled in a prevalent case-control study on 5 dairies in Tulare County, California. Dairy herd sizes varied between 800 and 3,600 milking cows. Enrollment occurred between September and December of 2016 and between April and July of 2017. Enrollment criteria for calves were weaned dairy calves housed in group pens with lock-up capabilities and either Jersey or Holstein breed. The study was approved by the University of California, Davis, Institutional Animal Care and Use Committee (study protocol \#19623).

\section{Farm Visits}

On study days, a veterinarian visited a participating dairy herd and observed 1 or 2 pens for the presence of calves displaying lethargy, depression, or separation from the group for about 10 to $15 \mathrm{~min}$. In addition to these sick-looking calves, randomly chosen calves were picked until a total of 8 to 10 calves were identified. A random number list was generated in a spreadsheet (Excel, Microsoft, Redmond, WA). The first calf observed whose eartag number ended in the next digit on the randomized list was selected as the next random calf. Presence or absence of the following clinical signs were recorded for all enrolled animals: nasal discharge, ocular discharge, cough, abnormal respiration (increased respiratory effort or respiratory rate higher than the average of the calves evaluated in the same pen on that day), depression (not bright, alert, or responsive), sunken eyes (visible gap between the lower eyelid margin and the globe), drooped ears or head tilt, separation from the group, lack of interest in feed (not walking up to the feedbunk when feed is delivered), lack of grooming (accumulation of dried, crusted dirt on haircoat), and low body condition compared with penmates (corresponding to a score of 2 or lower on a scale of 1-5). Calves with suspected active infectious bovine keratoconjunctivitis based on the presence of an active central corneal ulcer were not included in 
the study to avoid confounding by ocular discharge from this condition. Time and air temperature at start and end of visit were recorded. Visits were intended to take place before feed was delivered to calves. On some study days, calves were already locked up at arrival or feed had already been delivered so that calves could not be observed free in the pen before enrollment. Calves were restrained in typical head locks at the feed bunk, mostly after fresh feed was delivered. Rectal temperatures, respiratory rates, and heart rates were recorded. The calves' lung fields were ausculted for any abnormal lung sounds including wheezes, crackles, pleural friction, or increased bronchial sounds. Preparation for thoracic ultrasound examination included clipping of a calf's lateral thorax on both sides in a triangle bounded by the olecranon, the caudal angle of the scapula, and the transverse process of the 10th thoracic vertebra, and the skin moistened with $70 \%$ isopropyl alcohol. A portable ultrasound machine (Ibex Pro, E.I. Medical Imaging, Loveland, CO) with an 8-5 $\mathrm{MHz} 66 \mathrm{~mm}$ multifrequency linear transducer was used to scan lung parenchyma visible in intercostal spaces from 10 to 1 on the right and from 10 to 2 on the left for the following lesions: consolidation, abscess, pleural effusion, or comet tails. In older calves, the most cranial intercostal spaces were not always accessible due to increased muscle mass. Depth was set to $87 \mathrm{~mm}$ and gain was set to automatic. The veterinarian performing the ultrasound had several years of experience in livestock reproductive ultrasonography and had received training in thoracic ultrasonography of calves. Two farms had no corral fencing behind the lockup stanchions, and only calves that walked up to the feed bunk and became restrained after placing their heads through the stanchions could be enrolled. The preferential inclusion of calves into the study that walked up to feed led to the decision that "lack of interest in feed" could not be used as a predictor. Calves that could not be handled safely due to demeanor or that were at risk of injury from struggling in the headlocks were not enrolled.

\section{Case Definition}

Case status as a BRD positive calf was determined by thoracic ultrasound and auscultation interpreted in parallel (i.e., a positive diagnosis on at least one of the tests was sufficient to declare the calf as BRD positive; Love et al., 2016; Ollivett and Buczinski, 2016). Criteria for the diagnosis of BRD were detection of abnormal lung sounds or focal or extensive consolidation or abscesses of at least one lesion $2 \mathrm{~cm}$ width $\times$ $2 \mathrm{~cm}$ depth, or any amount of pleural effusion. Comet tails and smaller, discreet areas of consolidation were noted, but no diagnosis of BRD was made solely based on the presence of comet tails or a lobular pattern of consolidation (Ollivett and Buczinski, 2016).

\section{Statistical Analysis}

Sample Size. A minimum sample size of 575 calves was calculated as sufficient to be able to detect odds ratios (OR) of at least 2.0 for the presence of clinical signs in cases versus non-cases with a power of 0.80 and $\alpha$ at 0.05 assuming a prevalence of $20 \%$ and an exposure proportion of $20 \%$.

Model Development. All analyses were performed with a commercial software package (Stata 14.2, Stata Corp., College Station, TX). The level of significance for all analyses was $P<0.05$. The data were split randomly 80:20, and data exploration, univariable, and multivariable analysis for model development was performed on $80 \%$ of the data. Candidate models were then evaluated in 3-fold cross-validation (Lever and Altman, 2016) in which the entire data set was randomly split into 4 sets and testing of model performance was accomplished with part of the data not used for model training or validation (Figure 1).

Random 80:20 Data Split. A random number function in Excel was used to assign random numbers between 0 and 1 to all enrolled calves by dairy. The $20 \%$ of the calves from each dairy with the largest random numbers were set aside. The data sets comprising 80 and $20 \%$ of the data, respectively, were compared with Pearson's chi-squared tests for differences in cases, breeds, season, or sex, and by linear regression for differences in age. Sampling probability weights for enrolled calves $\left(\mathrm{w}_{\mathrm{ijk}}=\right.$ sampling probability weight for the ith calf in the jth pen on the kth day) were assigned as follows: clinically apparent cases (i.e., those selected for evaluation because they appeared sick) were assigned a weight of $1\left(\mathrm{w}_{\mathrm{ijk}}=1\right)$, and calves that were randomly identified and enrolled were assigned a weight equal to the inverse of the sampling fraction $\left(\mathrm{w}_{\mathrm{ijk}}=1 / \mathrm{f}_{\mathrm{jk}}\right)$. The sampling fraction for each location on a day $\left(\mathrm{f}_{\mathrm{jk}}\right)$ was defined by the proportion of calves per pen that were randomly sampled on that day, corrected for the rate of sampling of clinically apparent cases in that pen (Figure 2). Overall, the largest weights were trimmed to the 99th percentile of sampling weights to avoid using highly influential weights (Lavallee and Beaumont, 2015).

The list of predictors included all clinical signs recorded that are observable without handling the calf. Possible confounders included age, breed and sex of calf, season (spring or fall), and month and mean ambient temperature during the visit. The range in air temperature (24-h ambient temperature range) in degrees Celsius for the 24-h period preceding the 
visit as measured at the closest weather station in the National Oceanic and Atmospheric Administration's network (NOAA, 2018) was evaluated as a predictor as well as a confounder in separate models. Box-Tidwell tests for the continuous variables rectal temperature, mean ambient temperature during the visit, ambient temperature range in the $24 \mathrm{~h}$ before the visit, and age in days were performed to test for the assumption of linearity of independent variables related to the log odds (Box and Tidwell, 1962).

Survey-weighted univariable generalized linear mixed models with calf as the unit of analysis, BRD diagnosis as the outcome, and dairy as the random effect were performed for all predictors and confounders. Predictors were subsequently offered in forward selection to a multivariable model one at a time in order of increasing $P$-value in univariable analysis, with variables with $P<0.05$ in the multivariable model allowed to remain in the model. Two-way interaction variables for rectal temperature with season, month, mean ambient temperature during the visit, or ambient temperature range during the $24 \mathrm{~h}$ preceding the visit were tested for significance. Similarly, 2-way interactions of respiratory rate or abnormal respiration with ambient temperature were tested for significance. The variables age, sex, breed, mean ambient temperature during the visit, ambient temperature range in degrees Celsius for the 24-h period preceding the visit were assessed for confounding by adding them to the model one at a time and observing changes in OR. Variables that changed OR by more than $20 \%$ were considered confounders.

3-Fold Cross-Validation. Figure 1 graphically depicts the 3 -fold cross-validation process. The data set was split randomly into 4 equal groups balanced by season and diagnosis. Group 4 was set aside for testing. Survey-weighted groups of observations were compared with Pearson's chi-squared tests for differences in proportions of cases, breeds, season or sex, and by linear regression for differences in age. Three rounds of model training and validation were performed, each time using 2 of the 3 data sets for training and 1 for validation. Points were assigned to each clinical sign based on its regression coefficient in a model (Moons et al., 2002). Scores were based on doubled regression

I. Random data split into four
sets maintaining class
balance by season and
diagnosis
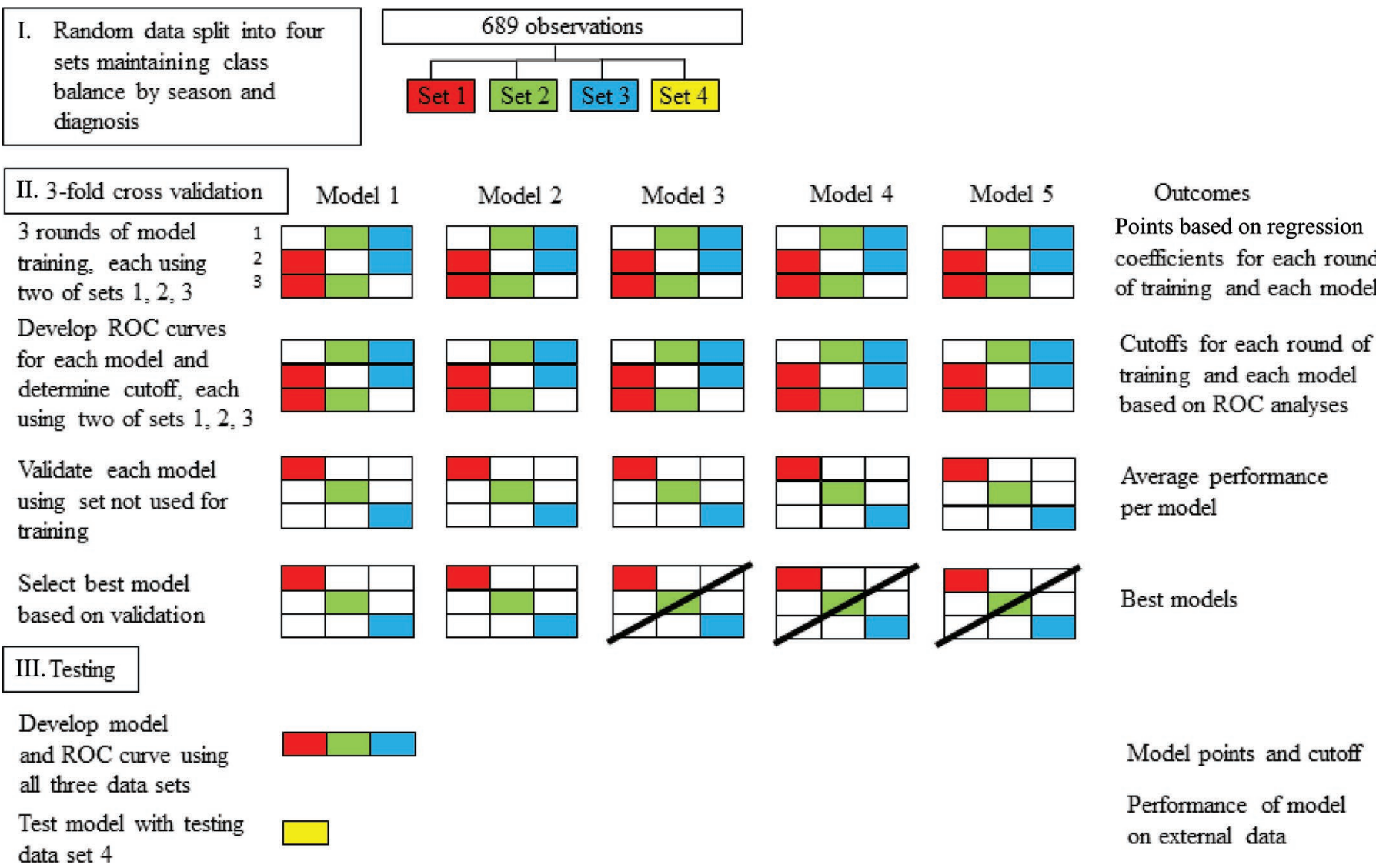

Figure 1. Flowchart of data in 3-fold cross-validation for development of a scoring system for bovine respiratory disease in a sample of 689 weaned dairy calves on 5 dairies in the San Joaquin Valley in California. ROC $=$ receiver operating characteristic. 
coefficients with all regression coefficients before doubling between 0 and $<0.75$ getting assigned a score of 1 , those between 0.75 and $<1.25$ getting assigned a score of 2 , those between 1.25 and $<1.75$ getting assigned a score of 3 and so on. Negative regression coefficients were assigned a score of 0 . In this manner, all observations in the 3 sets were used for model development and validation (Lever and Altman, 2016). A receiver operating characteristic (ROC) curve was used to determine the optimum cut-point for a positive score during each round of model training. Instead of probability weights, frequency weights were used in creating ROC curves due to software constraints. Model performance parameters were calculated based on this cutoff using the data set aside for validation during each round.

Model performance for each of 3 rounds of cross-validation was assessed based on the clinical scores assigned in terms of sensitivity, specificity, F1 score [harmonic average of sensitivity and positive predictive value $(2$ $\times$ sensitivity $\times$ positive predictive value)/(sensitivity + positive predictive value)], classification accuracy (percent of correct predictions), balanced accuracy (average of sensitivity and specificity), Matthew's correlation coefficient (MCC; correlation coefficient between observed and predicted binary classifications between -1 and +1 with +1 representing a perfect prediction) and area under the ROC curve (AUC; Powers, 2007; Boughorbel et al., 2017). Sensitivity was calculated as screening sensitivity for all calves in the data set as well as diagnostic sensitivity, which included only the sicklooking calves (i.e., the calves not randomly selected; Love et al., 2016). After all the models were validated, the average performance scores for the competing models were compared and the best model selected based on performance scores and parsimony. Regression coefficients for the model selected in 3-fold cross-validation were determined by running the analysis using data sets 1 through 3 . The cutoff maximizing sensitivity and specificity obtained from the ROC curve for points assigned based on regression coefficients from the model using data sets 1 through 3 was used to determine score positive and negative calves. Model performance was tested with data set 4 , which had not been used for model development.

Testing in Series with Rectal Temperature. Calves with a positive test score based on points assigned in the model chosen in 3-way cross-validation were further tested in series for fever, which was defined as a rectal temperature $\geq 39.2^{\circ} \mathrm{C}$, the cutoff defined as a fever in the California BRD scoring system for preweaned calves (Love et al., 2014). Scoring system performance after this final classification was assessed again using the same metrics as described above.

\section{RESULTS}

Of 689 calves enrolled in the study, 403 were Holsteins and 286 were Jerseys, 673 were female and 16 were male, 311 were enrolled in the fall (September through December), and 378 were enrolled in the spring (April through July). Calves were between 45 and 324 d old with a mean of $149 \pm 1.4(\mathrm{SE})$ d. The 5 dairies contributed 197, 238, 94, 83, and 77 calves each. A total of 89 calves were selected because they appeared sick based on displaying lethargy, depression, or separation from the group, and 600 were randomly selected. Bovine respiratory disease was diagnosed in $238(34.5 \%)$ of all enrolled calves including $70(78.7 \%)$ of the sick-looking calves. Of the 238 cases, 28 were diagnosed based on auscultation only, 113 based on ultrasound only and 97 based on both auscultation and ultrasound results. The survey-weighted proportions of calves with a rectal temperature $\geq 39.2^{\circ} \mathrm{C}$ by mode of diagnosis was $84.1 \%$ [95\% CI $(43.6,97.3)]$ for diagnosis by auscultation only, $83.9 \%$ [95\% CI $(75.2,89.9)]$ for diagnosis by ultrasound only, and $82.4 \%$ [95\% CI $(70.6,90.1)]$ for diagnosis by

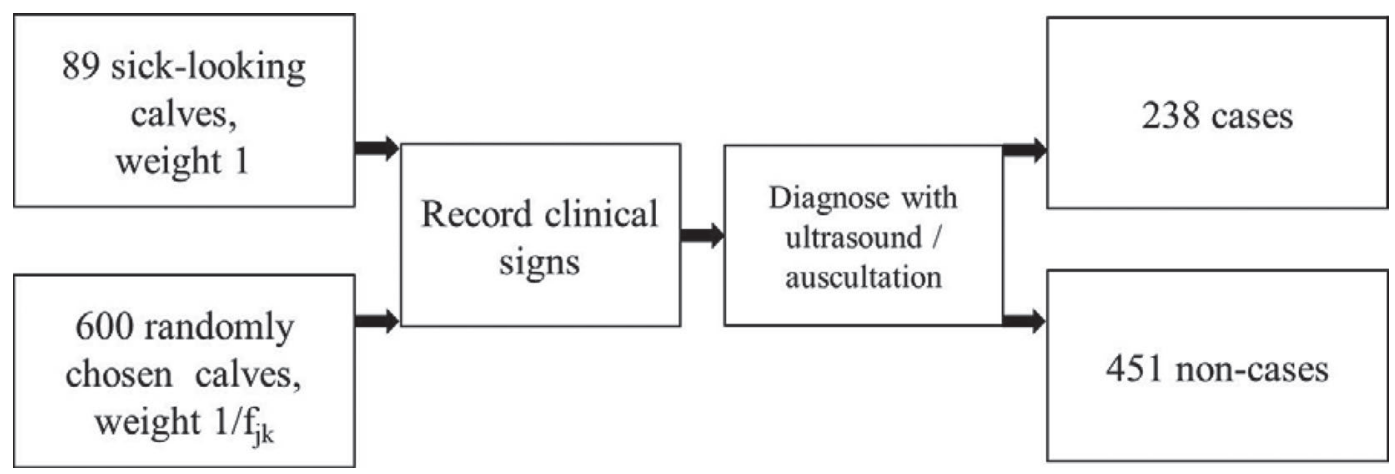

Figure 2. Flowchart of study design for development of a scoring system for bovine respiratory disease in a sample of 689 weaned dairy calves on 5 dairies in the San Joaquin Valley in California. $\mathrm{f}_{\mathrm{jk}}=$ sampling fraction for each location on a day. 
either modality. After survey adjustment, prevalence as diagnosed by thoracic ultrasound and auscultation in all calves was $26.3 \% \pm 1.9$ and $78.6 \% \pm 4.5$ in sick-looking calves. Reviewing daily maximum and minimum ambient temperatures measured at the local airport (Fresno Yosemite International Airport) and recorded by the National Oceanographic and Atmospheric Administration (NOAA, 2018) for the study period showed the following breakdown of study days during the study period where the 24-h ambient temperature range was $>15^{\circ} \mathrm{C}\left(27^{\circ} \mathrm{F}\right)$ : September, 2016: 9 of $11 \mathrm{~d}$; October, 2016: 10 of 31 d; November, 2016: 5 of 30 d; December, 2016: 0 of 8 d; April, 2016:4 of 20 d; May, 2016: 0 of 31 d; June, 2016: 22 of 30 d; July, 2016: 27 of 28 d. Of 9 calves with missing rectal temperatures, 8 were observed on the same day at the same farm due to failure of the temperature probe that day. Three calves had missing data on age and were eliminated from models that controlled for age as a confounder.

\section{0:20 Split}

The random 80:20 split resulted in 551 calves for model development. No significant differences were observed between calves used or not used for model development in terms of breed $(P=0.69)$, sex $(P=0.53)$, season $(P=0.41)$, age $(P=0.22)$, or proportion of calves diagnosed with $\mathrm{BRD}(P=0.62)$. The continuous variable for rectal temperature did not pass the test for the assumption of linearity of independent variables related to the log odds (Box-Tidwell test, $P<0.001$ ), but the variables for mean ambient temperature during the visit $(P=0.22)$, ambient temperature range in the past $24 \mathrm{~h}(P=0.23)$, and age in days $(P=0.86)$ did. Rectal temperature was therefore used as a dichotomous variable. Survey-weighted proportions of cases and noncases and survey-weighted univariable OR with dairy as random effect in the set used for model development are as follows: Nasal discharge was observed in $27.2 \%$ \pm 4.2 cases and $20.7 \% \pm 2.8$ non-cases with an OR of 1.41 [95\% CI: $(0.72,2.73), P=0.32]$. Ocular discharge was noted in $27.2 \% \pm 4.1$ of cases and $20.0 \% \pm 2.6$ non-cases with an OR of 1.39 [95\% CI: $(0.99,1.97), P$ $=0.06]$. Cough was recorded in $19.9 \% \pm 3.1$ of cases and $6.2 \% \pm 1.3$ of non-cases with an OR of 3.54 [95\% CI $(2.06,6.07), P<0.001]$. Abnormal respiration was seen in $58.6 \% \pm 4.4$ of cases and $45.1 \% \pm 3.2$ of noncases, resulting in an OR of 1.75 [95\% CI $(1.17,2.63)$, $P=0.006]$. Depression was observed in $4.3 \% \pm 1.6$ of cases and $0.6 \% \pm 0.4$ of non-cases, leading to an OR of 6.06 [95\% CI $(1.89,19.44), P=0.002]$. Sunken eyes were noted in $2.3 \% \pm 1.2$ of cases and $0.1 \% \pm 0.07 \%$ of non-cases for an OR of 11.62 [95\% CI $(2.71,49.85), P=$
0.001]. A head tilt or drooped ears were seen in $3.6 \% \pm$ 1.5 of cases and $0.6 \% \pm 0.3$ of non-cases, resulting in an OR of 3.72 [95\% CI $(1.77,7.83), P=0.01]$. Separation from the group was observed in $1.2 \% \pm 0.4$ of cases and $0.7 \% \pm 0.4$ of non-cases and the OR was 1.47 [95\% CI $(0.28,7.83), P=0.653]$. Lack of grooming was noted in $2.1 \% \pm 1.1$ of cases and $2.7 \% \pm 1.1$ of non-cases and the OR for this variable was $0.80[95 \%$ CI $(0.39,1.68)$, $P=0.56]$. A low body condition was seen in $15.1 \% \pm$ 3.4 of cases and $2.0 \% \pm 0.7$ of non-cases, and the OR for low body condition was 9.85 [95\% CI $(4.97,19.55)$, $P<0.001]$.

\section{Multivariable Model}

The model with the best fit from the selection process included variables for sunken eyes, low body condition, abnormal respiration, and cough, as well as the 24-h ambient temperature range and the confounders age and breed, which, when added to the model, changed the OR of at least one of the predictors by at least $20 \%$. The variable for mean ambient temperature during the visit was forced into the model to adjust for climatic conditions during the study period. There was no evidence for confounding by sex, season, or study month. The 2-way interactions of rectal temperature $>39.4^{\circ} \mathrm{C}\left(103.0^{\circ} \mathrm{F}\right)$ with season, month, mean ambient temperature during the visit, or ambient temperature difference during the last $24 \mathrm{~h}$ were not significant. A cutoff of $39.4^{\circ} \mathrm{C}\left(103.0^{\circ} \mathrm{F}\right)$ was chosen based on results from univariable analysis. Likewise, 2-way interactions of respiratory rate or abnormal respiration with ambient temperature were not significant. The estimated coefficients and scores assigned to each variable for models 1 and 2 are summarized in Table 1. Model 1 uses the 24-h ambient temperature range as a predictor, hence specified as a categorical variable to indicate whether or not the temperature range exceeded $15^{\circ} \mathrm{C}$ $\left(27^{\circ} \mathrm{F}\right)$. The cutoff of $15^{\circ} \mathrm{C}\left(27^{\circ} \mathrm{F}\right)$ is based on results from univariable analysis. In model 2 , the 24 -h ambient temperature range is included to adjust for the biological effect of temperature and hence was specified as a continuous variable.

\section{3-Fold Cross-Validation}

The 4-way data split balanced by season and BRD diagnosis resulted in group 1 with 171 total and 59 BRD diagnosis positive observations, group 2 with 173 total and 61 BRD diagnosis positive observations, group 3 with 171 total and 59 BRD-positive observations, and group 4 with 174 total and 59 BRD-positive observations. There were no differences between groups 
in terms of BRD-positive calves $(P=0.58)$, breeds $(P$ $=0.75)$, sex $(P=0.25)$, seasons $(P=0.65)$, or age $(P$ $=0.18)$.

Five models were tested using each of the 3 data sets from the 3 -fold cross-validation. Model 1 consisted of variables in the final model from the 80:20 data split including the 24-h ambient temperature range and possible confounders. Model 2 contained the same clinical predictors as model 1; however, 24-h ambient temperature range was not assigned any points and was used as a continuous variable to adjust for its biological effect. This model was tested to compare its performance to model 1 and because eliminating the temperature range would simplify field application of a scoring system from such a model. Model 3 contained the same predictors as model 2 in addition to the variables for nasal and ocular discharge. Nasal and ocular discharge were important predictors in the scoring system developed for preweaned calves and are easily recognized, hence the usefulness of these predictors was tested in this model. Model 4 assigned points based on the scoring system previously developed for preweaned calves by our group (Love et al., 2014), to assess whether this scoring system is also appropriate for weaned calves and, therefore, was not based on model development. Finally, model 5 contained forced-in variables for the same clinical signs as those making up the scoring system for preweaned calves but using coefficients, and hence scores, obtained from model training, and a cutoff for a positive rectal temperature score based on model fit using the current study data.

The estimated coefficients for models $1,2,3$, and 5 developed with data sets 1,2 , and 3 are summarized in Tables 2, 3, 4, and 5, respectively. In model 1, which contained the significant variables for clinical signs and 24-h ambient temperature range in the final model resulting from the 80:20 split, cough and low body condition were significant predictors in all 3 rounds of model training, sunken eyes was a significant predictor in 2 rounds of model training, abnormal respiration was a significant predictor in 1 round of model training, and the 24-h ambient temperature range was marginally significant in 2 out of 3 rounds of model training. In model 2, which contained the same variables as model 1 except the 24-h ambient temperature range was used as a continuous variable, low body condition and cough were significant predictors in all 3 rounds of model training, sunken eyes was a significant predictor in 2 rounds, and abnormal respiration was only significant in 1 round of model training. In model 3, which included clinical signs nasal discharge and ocular discharge in addition to all variables in model 2 , only low body condition and cough were significant predictors in all 3 rounds of model training, sunken eyes was a significant predictor in 2 rounds of model training, abnormal respiration and nasal discharge were significant predictors in 1 round of model training, and ocular discharge was never a significant predictor. For model 4, which was

Table 1. Generalized linear mixed models with a logit link modeling the association between presence of clinical signs and bovine respiratory disease diagnosed by thoracic ultrasound and auscultation in parallel in 542 weaned dairy calves on 5 dairies in California's San Joaquin Valley ${ }^{1}$

\begin{tabular}{|c|c|c|c|c|c|c|}
\hline Variable & \multicolumn{3}{|c|}{ Model 1} & \multicolumn{3}{|c|}{ Model 2} \\
\hline No & Referent & & & & & \\
\hline Yes & 0.61 & 0.25 & 0.02 & - & - & - \\
\hline \multicolumn{7}{|l|}{ Sunken eyes } \\
\hline Absent & Referent & & & Referent & & \\
\hline \multicolumn{7}{|l|}{ Low body condition } \\
\hline Absent & Referent & & & Referent & & \\
\hline Present & 2.26 & 0.43 & $<0.001$ & 2.21 & 0.40 & $<0.001$ \\
\hline \multicolumn{7}{|l|}{ Abnormal respiration } \\
\hline Absent & Referent & & & Referent & & \\
\hline Present & 0.63 & 0.27 & 0.02 & 0.63 & 0.27 & 0.02 \\
\hline \multicolumn{7}{|l|}{ Cough } \\
\hline None & Referent & & & Referent & & \\
\hline Mean ambient temperature during visit in ${ }^{\circ} \mathrm{C}$ & 0.010 & 0.02 & 0.69 & 0.004 & 0.02 & 0.86 \\
\hline 24 -h ambient temperature range in ${ }^{\circ} \mathrm{C}$ & - & - & - & 0.08 & 0.02 & $<0.001$ \\
\hline Intercept & -1.21 & 0.20 & $<0.001$ & -2.04 & 0.18 & $<0.001$ \\
\hline
\end{tabular}

\footnotetext{
${ }^{1}$ The data set consisted of $80 \%$ randomly selected observations of a total of 689 observations.
} 
Table 2. Model 1: generalized linear mixed models with a logit link modeling the association between presence of clinical signs and bovine respiratory disease diagnosed by thoracic ultrasound and auscultation in parallel developed in 3 rounds of cross-validation in 515 weaned dairy calves on 5 dairies in California's San Joaquin Valley ${ }^{1}$

\begin{tabular}{|c|c|c|c|c|c|c|c|c|c|}
\hline Variable & $\beta_{1}$ & $\operatorname{SE}\left(\beta_{1}\right)$ & $P$-value & $\beta_{2}$ & $\operatorname{SE}\left(\beta_{2}\right)$ & $P$-value & $\beta_{3}$ & $\mathrm{SE}\left(\beta_{3}\right)$ & $P$-value \\
\hline \multicolumn{10}{|l|}{$\begin{array}{l}24 \text {-h ambient temperature } \\
\text { range }>15^{\circ} \mathrm{C}\end{array}$} \\
\hline No & Referent & & & Referent & & & Referent & & \\
\hline Yes & 0.20 & 0.16 & 0.22 & 0.66 & 0.35 & 0.06 & 0.51 & 0.37 & 0.053 \\
\hline \multicolumn{10}{|l|}{ Sunken eyes } \\
\hline Absent & Referent & & & Referent & & & Referent & & \\
\hline Present & -0.54 & 0.79 & 0.50 & 2.40 & 0.36 & $<0.001$ & 2.35 & 0.87 & 0.007 \\
\hline \multicolumn{10}{|l|}{ Low body condition } \\
\hline No & Referent & & & Referent & & & Referent & & \\
\hline Yes & 2.75 & 0.60 & $<0.001$ & 2.64 & 0.31 & $<0.001$ & 2.10 & 0.71 & 0.003 \\
\hline \multicolumn{10}{|l|}{ Abnormal respiration } \\
\hline Absent & Referent & & & Referent & & & Referent & & \\
\hline Present & 0.40 & 0.35 & 0.26 & 0.70 & 0.30 & 0.02 & 0.22 & 0.21 & 0.30 \\
\hline \multicolumn{10}{|l|}{ Cough } \\
\hline None & Referent & & & Referent & & & Referent & & \\
\hline Any & 0.98 & 0.39 & 0.01 & 0.84 & 0.40 & 0.04 & 0.97 & 0.12 & $<0.001$ \\
\hline Calf age in days & -0.007 & 0.003 & 0.02 & -0.01 & 0.004 & 0.002 & -0.008 & 0.002 & 0.006 \\
\hline \multicolumn{10}{|l|}{ Calf breed } \\
\hline Holstein & Referent & & & Referent & & & Referent & & \\
\hline Jersey & 0.96 & 0.21 & $<0.001$ & 0.47 & 0.18 & 0.01 & 0.51 & 0.15 & $<0.001$ \\
\hline $\begin{array}{l}\text { Mean ambient temperature } \\
\text { during visit in }{ }^{\circ} \mathrm{C}\end{array}$ & -0.02 & 0.03 & 0.55 & 0.002 & 0.04 & 0.97 & -0.01 & 0.02 & 0.61 \\
\hline Intercept & -0.74 & 0.50 & 0.14 & -0.37 & 0.73 & 0.61 & -0.37 & 0.36 & 0.30 \\
\hline
\end{tabular}

${ }^{1} \beta_{1}, \beta_{2}$, and $\beta_{3}$ refer to the regression coefficients in each of 3 rounds of cross-validation.

based on the scoring system developed for preweaned dairy calves, no training was performed because the same variables and points determined in the preweaned system, including a rectal temperature cutoff value of $\geq 39.2^{\circ} \mathrm{C}$, were assigned. Finally, model 5 , where variable selection was also based on the scoring system in preweaned calves, but with coefficients assigned based on model training and the cutoff for a fever was selected at a rectal temperature of $\geq 39.4^{\circ} \mathrm{C}$, cough was a significant predictor in all 3 rounds of model training, rectal temperature was a significant predictor in 2 of the 3 rounds of model training, head tilt or ear droop was a significant predictor in 1 round of model training, and abnormal respiration, nasal discharge, and ocular discharge were never significant predictors.

\section{Model Validation}

Results for the average performance of models in 3 rounds of model validation are outlined in Table 6. Model 1 was chosen based on parsimony and performance with the highest screening sensitivity after model 2 (64.0 and $70.0 \%$, respectively), the highest F1 score (0.49), AUC (68.7\%), and balanced accuracy (65.5\%). Model 2 was chosen as a simplified version of model 1 because it does not require knowledge of ambient temperature range in the $24 \mathrm{~h}$ before scoring. Running model 1 with data sets 1,2 , and 3 resulted in the following coefficients: sunken eyes: $\beta=1.91$ (SE:
$0.71, P=0.006)$, low body condition: $\beta=2.48$ (SE: $0.23, P<0.001$ ), abnormal respiration: $\beta=0.42$ (SE: $0.22, P=0.063$ ), cough: $\beta=0.90$ (SE: $0.22, P<0.001$ ), and 24 -h ambient temperature range $>15^{\circ} \mathrm{C}: \beta=0.42$ (SE: 0.09, $P<0.001$ ). Performance metrics for models 1 and 2 resulting from 3 -fold cross-validation with and without a second-tier test with rectal temperature are shown in Table 7. Model 1 without rectal temperature had a screening sensitivity of $77.0 \%$ (95\% CI: 61.9, 87.3 ), diagnostic sensitivity of $100 \%$, and specificity of $61.9 \%$ (95\% CI: 50.8, 72.0). Second-tier screening of test score positive calves by applying a second criterion to count as positive if the calf also had a rectal temperature of $\geq 39.2^{\circ} \mathrm{C}$ resulted in a screening sensitivity $64.8 \%$ (95\% CI: $49.1,77.9$ ), diagnostic sensitivity $76.9 \%$ (95\% CI: 42.8, 93.7), and specificity $76.7 \%$ (95\% CI: $67.1,84.1)$. The resulting scoring system assigns the following scores: sunken eyes: 4 points, low body condition: 5 points, abnormal respiration: 1 point, cough: 2 points, 24-h ambient temperature range $>15^{\circ} \mathrm{C}\left(27^{\circ} \mathrm{F}\right)$, 1 point. A calf is score suspect for BRD if it has a score of at least 2 points. If suspect calves have a rectal temperature $\geq 39.2^{\circ} \mathrm{C}$, they are score positive for BRD. The alternative model with scores adjusted for 24-h ambient temperature range, but which does not require knowledge of it, assigns the same scores for the clinical signs sunken eyes, low body condition, abnormal respiration, or cough, but the calf is score positive, if any of the clinical signs are observed. Following second-tier screening, 
Table 3. Model 2: generalized linear mixed models with a logit link modeling the association between presence of clinical signs and bovine respiratory disease diagnosed by thoracic ultrasound and auscultation in parallel developed in 3 rounds of cross-validation in 515 weaned dairy calves on 5 dairies in California's San Joaquin Valley ${ }^{1}$

\begin{tabular}{|c|c|c|c|c|c|c|c|c|c|}
\hline Variable & $\beta_{1}$ & $\operatorname{SE}\left(\beta_{1}\right)$ & $P$-value & $\beta_{2}$ & $\mathrm{SE}\left(\beta_{2}\right)$ & $P$-value & $\beta_{3}$ & $\operatorname{SE}\left(\beta_{3}\right)$ & $P$-value \\
\hline \multicolumn{10}{|l|}{ Sunken eyes } \\
\hline Absent & Referent & & & Referent & & & Referent & & \\
\hline Present & -0.59 & 0.76 & 0.44 & 2.54 & 0.32 & $<0.001$ & 2.48 & 0.91 & 0.007 \\
\hline \multicolumn{10}{|l|}{ Low body condition } \\
\hline No & Referent & & & Referent & & & Referent & & \\
\hline Yes & 2.82 & 0.63 & $<0.001$ & 2.55 & 0.38 & $<0.001$ & 2.05 & 0.72 & 0.005 \\
\hline \multicolumn{10}{|l|}{ Abnormal respiration } \\
\hline None & Referent & & & Referent & & & Referent & & \\
\hline Any & 0.98 & 0.36 & 0.006 & 0.83 & 0.35 & 0.018 & 0.96 & 0.14 & $<0.001$ \\
\hline Calf age in days & -0.006 & 0.003 & 0.04 & -0.01 & 0.004 & 0.002 & -0.006 & 0.002 & 0.02 \\
\hline \multicolumn{10}{|l|}{ Calf breed } \\
\hline Holstein & Referent & & & Referent & & & & & \\
\hline Jersey & 1.00 & 0.20 & $<0.001$ & 0.45 & 0.18 & 0.012 & 0.53 & 0.14 & $<0.001$ \\
\hline
\end{tabular}

${ }^{1} \beta_{1}, \beta_{2}$, and $\beta_{3}$ refer to the regression coefficients in each of 3 rounds of cross-validation.

the model achieves the following performance: screening sensitivity: $84.2 \%$ (95\% CI: $69.7,92.5)$, diagnostic sensitivity: $100 \%$ and specificity: $45.7 \%$ (95\% CI: 35.2 ,
56.6). Figure 3 is a visual aid that can be used in the field for the scoring system relying on 24-h ambient temperature (model 1), the scoring system with scores

Table 4. Model 3: generalized linear mixed models with a logit link modeling the association between presence of clinical signs and bovine respiratory disease diagnosed by thoracic ultrasound and auscultation in parallel developed in 3 rounds of cross-validation in 515 weaned dairy calves on 5 dairies in California's San Joaquin Valley ${ }^{1}$

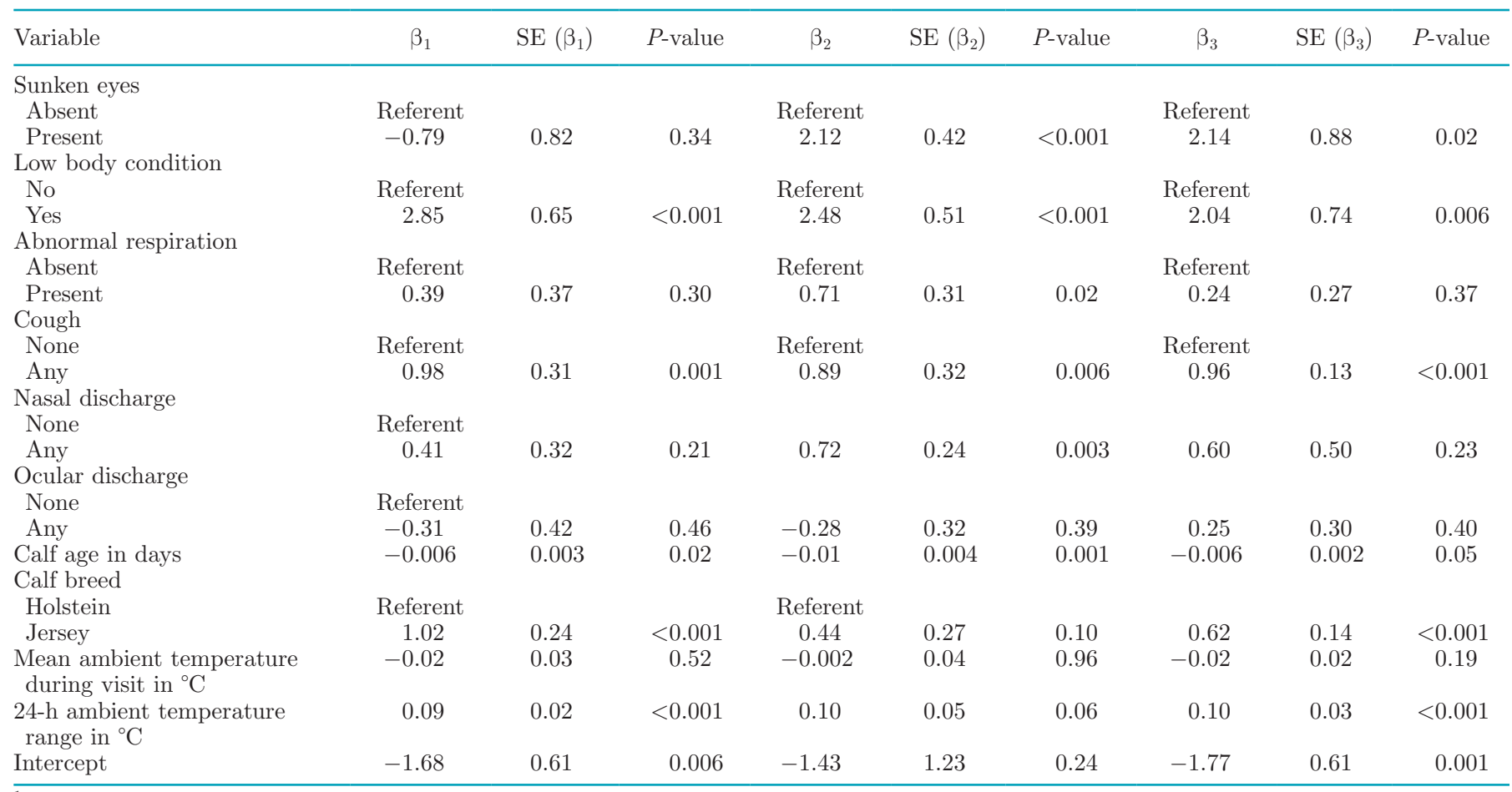

${ }^{1} \beta_{1}, \beta_{2}$, and $\beta_{3}$ refer to the regression coefficients in each of 3 rounds of cross-validation. 
Table 5. Model 5: generalized linear mixed models with a logit link modeling the association between presence of clinical signs and bovine respiratory disease diagnosed by thoracic ultrasound and auscultation in parallel developed in 3 rounds of cross-validation in 515 weaned dairy calves on 5 dairies in California's San Joaquin Valley ${ }^{1}$

\begin{tabular}{|c|c|c|c|c|c|c|c|c|c|}
\hline Variable & $\beta_{1}$ & $\operatorname{SE}\left(\beta_{1}\right)$ & $P$-value & $\beta_{2}$ & $\operatorname{SE}\left(\beta_{2}\right)$ & $P$-value & $\beta_{3}$ & $\mathrm{SE}\left(\beta_{3}\right)$ & $P$-value \\
\hline \multicolumn{10}{|c|}{ Abnormal respiration } \\
\hline Absent & Referent & & & Referent & & & Referent & & \\
\hline Present & 0.30 & 0.36 & 0.40 & 0.57 & 0.33 & 0.09 & 0.24 & 0.21 & 0.25 \\
\hline None & Referent & & & Referent & & & Referent & & \\
\hline Any & 1.25 & 0.23 & $<0.001$ & 1.11 & 0.27 & $<0.001$ & 1.24 & 0.12 & $<0.001$ \\
\hline \multicolumn{10}{|c|}{ Nasal discharge } \\
\hline \multicolumn{10}{|c|}{ Ocular discharge } \\
\hline Absent & Referent & & & Referent & & & Referent & & \\
\hline Present & 0.04 & 0.38 & 0.91 & -0.04 & 0.19 & 0.84 & 0.36 & 0.28 & 0.200 \\
\hline \multicolumn{10}{|c|}{ Rectal temperature } \\
\hline No & Referent & & & Referent & & & Referent & & \\
\hline Yes & 0.32 & 0.35 & 0.35 & 0.56 & 0.23 & 0.01 & 0.87 & 0.32 & $<0.007$ \\
\hline
\end{tabular}

${ }^{1} \beta_{1}, \beta_{2}$, and $\beta_{3}$ refer to the regression coefficients in each of 3 rounds of cross-validation.

adjusted for 24-h ambient temperature (model 2), and both models implemented with a second-tier screening by recording the calf's rectal temperature as a confirmatory step.

\section{DISCUSSION}

To our knowledge, this is the first report of a scoring system for BRD in weaned dairy calves using field data. Previous scoring systems were developed in preweaned dairy calves (Love et al., 2014; McGuirk and Peek, 2014) or in calves experimentally infected with 1 or 2 specific respiratory pathogens (Thomas et al., 1977; Verhoeff et al., 1985; Gershwin et al., 2011; Amrine et al., 2013). The presented scoring system identifies a unique set of clinical signs and environmental measures that allows dairy producers to identify weaned calves likely to benefit from BRD treatment and minimize or reduce unnecessary treatment in those that may not require it. The scoring system assesses 4 simple

Table 6. Results for the average performance of models in 3 rounds of model validation for the association between presence of clinical signs and bovine respiratory disease diagnosed by thoracic ultrasound and auscultation in parallel in 515 weaned dairy calves on 5 dairies in California's San Joaquin Valley

\begin{tabular}{|c|c|c|c|c|c|c|c|c|}
\hline Model $^{1}$ & $\begin{array}{c}\text { Screening } \\
\text { sensitivity } \\
(\%)\end{array}$ & $\begin{array}{l}\text { Diagnostic } \\
\text { sensitivity } \\
(\%)\end{array}$ & $\begin{array}{c}\text { Specificity } \\
(\%)\end{array}$ & $\begin{array}{c}\mathrm{AUC}^{2} \\
(\%)\end{array}$ & $\begin{array}{c}\text { F1 score } \\
(\%)\end{array}$ & $\begin{array}{c}\text { Classification } \\
\text { accuracy }^{4} \\
(\%)\end{array}$ & $\begin{array}{l}\text { Balanced } \\
\text { accuracy }^{5} \\
\quad(\%)\end{array}$ & $\begin{array}{l}\text { Matthew's } \\
\text { correlation } \\
\text { coefficient }^{6}\end{array}$ \\
\hline 1 & 64.0 & 91.8 & 63.8 & 68.7 & 49.0 & 66.0 & 65.5 & 0.32 \\
\hline 3 & 44.9 & 92.7 & 82.2 & 66.6 & 45.8 & 72.3 & 63.5 & 0.40 \\
\hline 4 & 45.4 & 73.9 & 75.9 & 64.8 & 42.1 & 66.0 & 60.7 & 0.37 \\
\hline 5 & 53.7 & 91.8 & 70.3 & 65.5 & 45.2 & 66.0 & 62.0 & 0.41 \\
\hline
\end{tabular}

${ }^{1}$ Model 1: 24-h ambient temperature range $>15^{\circ} \mathrm{C}\left(27^{\circ} \mathrm{F}\right)$, sunken eyes, low body condition, abnormal respiration, cough, calf age, breed, mean ambient temperature during visit; model 2: sunken eyes, low body condition, abnormal respiration, cough, calf age, breed, mean ambient temperature during visit, 24-h ambient temperature range; model 3: sunken eyes, low body condition, abnormal respiration, cough, nasal discharge, ocular discharge, calf age, breed, mean ambient temperature during visit, 24-h ambient temperature range; model 4: ocular discharge, nasal discharge, head tilt/ear droop, cough, abnormal respiration, rectal temperature $\geq 39.2^{\circ} \mathrm{C}$; model 5: ocular discharge, nasal discharge, head tilt, ear droop, cough, abnormal respiration, rectal temperature $\geq 39.4^{\circ} \mathrm{C}\left(103.0^{\circ} \mathrm{F}\right)$.

${ }^{2}$ Area under the curve.

${ }^{3}$ Harmonic average of sensitivity and positive predictive value $(2 \times$ sensitivity $\times$ positive predictive value $) /($ sensitivity + positive predictive value).

${ }^{4}$ Percent of correct predictions.

${ }^{5}$ Average of sensitivity and specificity.

${ }^{6}$ Correlation coefficient between observed and predicted binary classifications between -1 and +1 with +1 representing a perfect prediction. 
Table 7. Results for the performance of testing models for the association between presence of clinical signs and bovine respiratory disease diagnosed by thoracic ultrasound and auscultation in parallel in 174 weaned dairy calves on 5 dairies in California's San Joaquin Valley

\begin{tabular}{|c|c|c|c|c|c|c|c|}
\hline Model $^{1}$ & $\begin{array}{c}\text { Screening } \\
\text { sensitivity } \\
(\%)\end{array}$ & $\begin{array}{c}\text { Diagnostic } \\
\text { sensitivity } \\
(\%)\end{array}$ & $\begin{array}{l}\text { Specificity } \\
(\%)\end{array}$ & $\begin{array}{c}\text { F1 score } \\
(\%)\end{array}$ & $\begin{array}{c}\text { Classification } \\
\text { accuracy }^{3} \\
(\%)\end{array}$ & $\begin{array}{l}\text { Balanced } \\
\text { accuracy }^{4} \\
(\%)\end{array}$ & $\begin{array}{l}\text { Matthew's } \\
\text { correlation } \\
\text { coefficient }^{5}\end{array}$ \\
\hline 1 & 77.0 & 100 & 61.9 & 53.5 & 69.0 & 69.5 & 0.41 \\
\hline 2 & 84.2 & 100 & 45.7 & 49.2 & 62.6 & 65.0 & 0.44 \\
\hline 2 with rectal temperature & 70.5 & 76.9 & 62.6 & 50.5 & 64.9 & 66.5 & 0.30 \\
\hline
\end{tabular}

${ }^{1}$ Model 1: 24-h ambient temperature range $>15^{\circ} \mathrm{C}\left(27^{\circ} \mathrm{F}\right)$, sunken eyes, low body condition, abnormal respiration, cough, calf age, breed, mean ambient temperature during visit; model 2: sunken eyes, low body condition, abnormal respiration, cough, calf age, breed, mean ambient temperature during visit, 24-h ambient temperature range.

${ }^{2}$ Harmonic average of sensitivity and positive predictive value $(2 \times$ sensitivity $\times$ positive predictive value $) /($ sensitivity + positive predictive value).

${ }^{3}$ Percent of correct predictions.

${ }^{4}$ Average of sensitivity and specificity.

${ }^{5}$ Correlation coefficient between observed and predicted binary classifications between -1 and +1 with +1 representing a perfect prediction.

predictors: cough (2 points), abnormal respiration (1 point), sunken eyes (4 points), and a low body condition (5 points) with a 2-point cutoff for a BRD suspect score. Adding the predictor 24-h ambient temperature range $>15^{\circ} \mathrm{C}\left(27^{\circ} \mathrm{F} ; 1\right.$ point $)$ to the model increases its specificity, and hence should be considered if this information is readily available. The 3 -fold cross-validation and testing of the model with data not used for model development increased the model's internal validity. Furthermore, inclusion of a wide range of cases and ages, Jersey and Holstein breeds, spring and fall seasons, and 5 different herds in a field setting increase the study's external validity. The inclusion of secondtier testing based on rectal temperature increases the specificity of the scoring system, limiting unnecessary treatments of calves, which should help promote judicious use of antimicrobial drugs. The optional inclusion of the 24-h ambient temperature range preceding a visit increases specificity of the system. Adjusting for the mean ambient temperature during the visit allows for use of the system at climactic environments different than the one where it was developed. Adjusting for ambient temperature takes the variation in outcome that is predicted by this factor into account so that the effect of the clinical predictors on the outcome can be isolated with greater precision. In lieu of the reference standard necropsy, thoracic ultrasound and auscultation were used in parallel to increase the sensitivity of these antemortem tests for the detection of animals with clinical BRD.

\section{Clinical Signs Assessed}

Sunken eyes, low body condition, and abnormal respiration are signs that may be subjective and are of most value if evaluated relative to penmates rather than in isolation. In this study, only a few animals were categorized as having sunken eyes, but the sign was strongly correlated with BRD. Sunken eyes were seen in the most compromised calves, and a more generous assignment of what constitutes sunken may have led to different results. Sunken eyes or dehydration in preweaned calves may be more specific for diarrhea than respiratory disease; however, weaned calves are less likely to be affected by diarrhea, and thus, sunken eyes may be more specific for BRD during this life stage. Dehydration has been described and named as a sign in BRD in feedlot cattle (Snowder et al., 2006). Dehydrated animals due to BRD are severely affected during the acute stage of the disease and should alert the scorer to an acute outbreak if several such animals are seen.

The assignment of a low body condition was given to calves that were clearly different in condition from their penmates and were often not just thin but could be described as stunted in their growth. Low body condition is associated with chronic disease as the animal loses condition over the course of days or weeks. In feedlot steers, lung lesions at slaughter were associated with decreased ADG compared with animals with normal lungs at slaughter (Wittum et al., 1996). Low body condition is a sign that may have lower inter-rater agreement than signs such as the presence of a cough, and it should be assigned corresponding to a score of 2 or less on a 5-point scale for body condition. A scorer should become suspicious of a systemic long-term herd problem if multiple calves in different age groups score positive for low body condition.

Increased respiratory effort is typically easily recognized and often is associated with BRD (Gershwin et al., 2011). An increased respiratory rate may also be caused by BRD. Eberhart et al. (2017) observed the presence of an increased respiratory rate throughout a 7-d study period in Holstein calves experimentally 


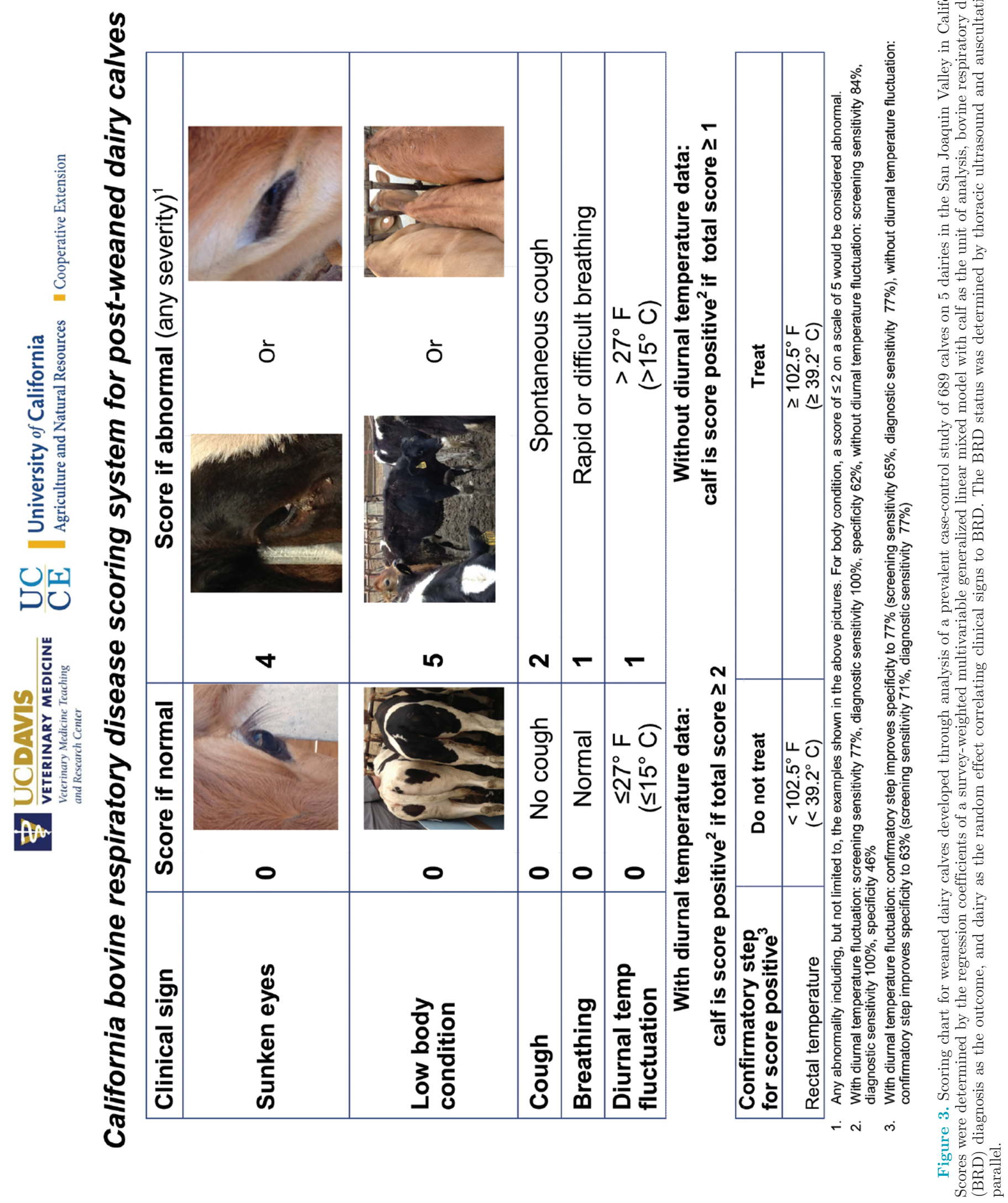


infected with Mannheimia haemolytica compared with a control group. However, the sign should only be considered positive after comparing the respiratory rate with that of other calves in the same pen. A fast respiratory rate can be caused by heat stress or excitement and thus may not be due to BRD. Difficult or labored breathing on the other hand is often correlated with severe disease. We chose not to separate the 2 signs for the sake of parsimony but realize that tachypnea and dyspnea may not reflect the same severity of disease.

Cough is easily recognized and correlated with BRD in preweaned calves (Love et al., 2014) but can often be observed while animals are eating, especially when feed is first delivered to a hungry group of animals. Excited animals that have increased physical activity, especially in open lots that can be dusty, are also often heard coughing. Hence cough should be evaluated in calves at a resting state. Because the scoring system is intended to be used while observing animals from afar, there should ideally be little interference due to excitation or environmental circumstances.

One of the clinical signs that is often used in BRD scoring systems is rectal temperature. We purposely tried to avoid the use of rectal temperature as a required component for every calf in our system for several reasons. Taking a rectal temperature requires handling and restraint of the calf, which is often impractical in weaned calves and could add stress to the handled calf as well as the penmates when attempting to restrain a calf in a group pen. In addition, body temperature may only remain elevated for a brief period during the course of BRD and therefore reliance on this sign may miss positive animals. Only calves that are suspect for BRD based on a positive score need to be handled with this system to minimize stress. We added a confirmatory step for score-positive calves that is based on a rectal temperature $\geq 39.2^{\circ} \mathrm{C}$ to increase specificity of the system. However, sensitivity dropped by approximately $13 \%$ after implementing this second-tier testing by rectal temperature. Holstein steers experimentally infected with Mannheimia haemolytica had significantly elevated rectal temperatures compared with a control group only during one time point ( $12 \mathrm{~h}$ postinfection) when measuring study animals twice daily for $7 \mathrm{~d}$ (Eberhart et al., 2017). In light of the research showing that rectal temperature may only be elevated in calves with BRD for a brief period of time, it is important to understand that implementing this confirmatory step is only useful if calves are monitored daily so sick calves are not misclassified as negative after their rectal temperature has returned to normal.

In a comprehensive review on the effects of temperature and humidity on animal diseases, Dennis (1986) concluded that sudden changes of weather may be more important than steady extremes of temperature and humidity, to which an animal may adapt. In a review of biosecurity and BRD, Callan and Garry (2002) also mentioned rapidly changing environmental temperatures as a predisposing factor for BRD. High temperature fluctuations are more often seen during certain times of the year than others and may pose a stressor to calves predisposing them to BRD. In the San Joaquin Valley, during the study period, wide-ranging temperature fluctuations were seen in September, October, June, and July, which may be different in other parts of the country. Daily ambient temperature fluctuations in the $24 \mathrm{~h}$ before the visit were significantly associated with BRD in the study animals, and although the variable did not qualify as a confounder based on change of magnitude of the effect measure odds ratio when added to the model or as an effect modifier for rectal temperature or abnormal respiration, it was deemed appropriate to add the variable to models 2 and 3 to adjust for climactic effects to BRD in addition to the variable for mean ambient temperature during the visit.

\section{Model Performance}

Besides screening sensitivity, which is calculated using all calves in the sample, we also determined diagnostic sensitivity, which is calculated on the subset of calves that appear sick based on depression, lethargy, or separation from the group. A screening sensitivity of $64.8 \%$ in model 1 and $70.5 \%$ in model 2 for all calves in the testing set after applying the secondary testing with rectal temperature is acceptable, considering that some of the cases seen were mild or may have been due to chronic lesions or were recovering. In the subset of sick-looking calves, the diagnostic sensitivity of the scoring systems is $76.9 \%$, capturing a higher proportion of diseased calves compared with all study calves. Given that rectal temperature may only be elevated during the first $12 \mathrm{~h}$ of the disease, we may have missed some diseased animals, as demonstrated by the decrease in sensitivity of the system once rectal temperature is added as a second tier. On the other hand, adding a rectal temperature as a second tier increased the specificity of $76.7 \%$ (from $61.9 \%$ ) for model 1 and $62.6 \%$ (from $45.7 \%$ ) for model 2 . However, such specificity means that if one were to treat all score-positive animals, a substantial number may not actually require treatment. It is possible that a longitudinal study may have led to a higher cutoff for rectal temperature for a BRD-positive animal and ultimately resulted in higher specificity. However, the advantage of the 2-tiered system is that only score-positive animals pulled out of a pen and restrained need to be tested for fever, and the decision to treat can be made immediately after 
checking the rectal temperature of a calf that triggered a positive score.

In addition to sensitivity and specificity, we also evaluated the F1 score, classification accuracy, balanced accuracy, AUC, and MCC of models. The F1 score is suggested by Lever and Altman (2016) as a metric for model selection after $\mathrm{k}$-fold cross-validation and is the harmonic average of sensitivity and positive predictive value. Classification accuracy is the percent of correct predictions made by the model. Balanced accuracy is the average of sensitivity and specificity of the model. The aforementioned metrics, though commonly used, may be biased by the underlying prevalence or case balance (Powers, 2007). However, MCC is defined in terms of true positives, true negatives, false positives, and false negatives and is robust to data imbalance for cases and controls. Values of MCC range from -1 to 1 , with 1 showing complete agreement, -1 complete disagreement, and 0 no correlation. Both MCC and AUC have been chosen as the elective metric in the US Food and Drug Administration-led initiative MAQC-II that aims to reach a consensus on the best practices for development and validation of predictive models for personalized medicine (Boughorbel et al., 2017). Of the models chosen in 3-fold cross-validation, model 1 had the highest AUC, and although it had the lowest MCC, it also had the highest F1 score.

\section{BRD Diagnosis}

In our study, we used results from thoracic ultrasound and auscultation as parameters associated with BRD cases. Recently, attention has been given to thoracic ultrasound for the diagnosis of BRD due to its ease of application in the field and ability to distinguish between upper and lower respiratory tract infections (Love et al., 2016; Ollivett and Buczinski, 2016). However, studies on the performance of thoracic auscultation and ultrasound show that neither test is a perfect reference standard for the diagnosis of BRD in calves. Sensitivity and specificity of ultrasound were estimated at 85 and 94\%, respectively, in one study (Rabeling et al., 1998) and 94 and $100 \%$ in a different study (Ollivett et al., 2015). Thoracic auscultation was found to have low sensitivity (5.9\%) when only classifying crackles, wheezes, pleural friction rubs, and absence of respiratory sounds as abnormal (Buczinski et al., 2014). A different study evaluated the incremental value of thoracic ultrasound over auscultation for diagnosis of $\mathrm{BRD}$ in preweaned calves and found a sensitivity of $72.9 \%$ and specificity of $53.3 \%$ for auscultation (Buczinski et al., 2016). The latter study included increased bronchial sounds as abnormal, which was also used as a criterion for a positive diagnosis in the present study along with crackles, wheezes, pleural friction rubs, and absence of respiratory sounds. Because weaned calves on dairies are not destined for slaughter in the short term, examining lung lesions at slaughter as a reference test was not an available method. Using both auscultation and ultrasound in parallel and including increased bronchial sounds as a criterion for a BRD diagnosis increased sensitivity but potentially lowered specificity of the reference tests. No absolute cutoff for BRD-positive animals diagnosed by thoracic ultrasound exists in the literature. According to one system suggested by Ollivett and Buczinski (2016), normal aerated lung with few comet tails, diffuse comet tail artifacts without consolidation, and small lobular lesions are most likely viral in nature. To exclude calves with lobular lesions from cases, a cutoff for the size of consolidation of $2 \times 2$ $\mathrm{cm}$ was chosen. Other criteria may have led to different results.

\section{Strengths and Limitations}

A strength of this study is the inclusion of a wide spectrum of cases into the sample of evaluated calves. By choosing calves at random from a pen, a wide spectrum of BRD cases including calves that had relatively mild disease were included in the sample. Spectrum bias exists when only extreme cases and healthy individuals are compared and leads to overestimation of test performance (Buczinski and O'Connor, 2016). Other strengths of the study are the inclusion of a wide range of ages, Jersey and Holstein breeds, and sampling of calves at multiple dairies and during different seasons. The 3-fold cross-validation technique allowed multiple data splits for model building, reducing the chance of an unlucky data split into training and validation sets that may have led to a model with poor prediction (Lever and Altman, 2016). Finally, testing the model on data not used for model training is the next best option to testing the model in a different population.

A longitudinal study design would have permitted to assess clinical signs in acute cases as well as the opportunity to exclude calves with chronic lung lesions on ultrasound or calves that already had received treatment. Another limitation is the fact that the person diagnosing the study calves was not blinded to the clinical signs present or the result of the other reference test, which could have led to diagnostic review bias (Buczinski and O'Connor, 2016). Ideally, an independent evaluator should have assigned a diagnosis based on recorded ultrasonograms or lung sounds without having seen the calf. However, employing blinded, independent evaluators was not possible due to time and resources available. A further limitation is the possibility of classification bias in this study, which is 
possible with an imperfect diagnostic test. As explained earlier, the reference standard necropsy in combination with hisopathological, microbiological, molecular, and biochemical testing methods was not an option for our study population. In addition, the study included considerably fewer male calves compared with females and was limited in geographic location and climate, and hence our findings may not be observed elsewhere to the extent reported here. Finally, few of the animals present were excluded from enrollment because their demeanor made it unsafe to examine them, possibly biasing the sample.

\section{CONCLUSIONS}

The clinical scoring systems for BRD in weaned dairy calves developed in this study allows quick and easy assessment of calves housed in group pens for BRD. In the system that includes the 24 -h ambient temperature range, the combination of any 2 signs or 1 of the signs cough, low body condition, or sunken eyes trigger a suspect score. In the system excluding 24-h ambient temperature fluctuation, any of the signs cough, low body condition, sunken eyes, or abnormal respiration trigger a suspect score. Treating only those calves with a rectal temperature $\geq 39.2^{\circ} \mathrm{C}$ may ensure the judicious use of antimicrobials, but cases may be missed if observations are made less frequently than once daily.

\section{ACKNOWLEDGMENTS}

The authors thank the dairy owners, their staff, and herd veterinarians for their participation. Funding for this project was provided by the University of California Division of Agriculture and Natural Resources, Competitive Grants Program (PI: S. Aly, Project 1753), USDA National Institute of Food and Agriculture (PI: S. Aly; Projects: CA-V-PHR-4041-H and CALVAH-360), and the Dairy Epidemiology Laboratory (Aly laboratory), School of Veterinary Medicine, University of California, Davis.

\section{REFERENCES}

Abutarbush, S. M., C. M. Pollock, B. K. Wildman, T. Perrett, O. C. Schunicht, R. K. Fenton, S. J. Hannon, A. R. Vogstad, G. K. Jim, and C. W. Booker. 2012. Evaluation of the diagnostic and prognostic utility of ultrasonography at first diagnosis of presumptive bovine respiratory disease. Can. J. Vet. Res. 76:23-32.

Amrine, D. E., B. J. White, R. Larson, D. E. Anderson, D. A. Mosier, and N. Cernicchiaro. 2013. Precision and accuracy of clinical illness scores, compared with pulmonary consolidation scores, in Holstein calves with experimentally induced Mycoplasma bovis pneumonia. Am. J. Vet. Res. 74:310-315. https://doi.org/10.2460/ ajvr.74.2.310.
Boughorbel, S., F. Jarray, and M. El-Anbari. 2017. Optimal classifier for imbalanced data using Matthews Correlation Coefficient metric. PLoS One 12:e0177678. https://doi.org/10.1371/journal.pone .0177678 .

Box, G. E. P., and P. W. Tidwell. 1962. Transformation of the independent variables. Technometrics 4:531-550.

Buczinski, S., G. Forte, D. Francoz, and A. M. Belanger. 2014. Comparison of thoracic auscultation, clinical score, and ultrasonography as indicators of bovine respiratory disease in preweaned dairy calves. J. Vet. Intern. Med. 28:234-242. https://doi.org/10.1111/ jvim. 12251.

Buczinski, S., J. Menard, and E. Timsit. 2016. Incremental value (Bayesian framework) of thoracic ultrasonography over thoracic auscultation for diagnosis of bronchopneumonia in preweaned dairy calves. J. Vet. Intern. Med. 30:1396-1401. https://doi.org/ 10.1111 /jvim.14361.

Buczinski, S., and A. M. O'Connor. 2016. Specific challenges in conducting and reporting studies on the diagnostic accuracy of ultrasonography in bovine medicine. Vet. Clin. North Am. Food Anim. Pract. 32:1-18. https://doi.org/10.1016/j.cvfa.2015.09.009.

Callan, R. J., and F. B. Garry. 2002. Biosecurity and bovine respiratory disease. Vet. Clin. North Am. Food Anim. Pract. 18:57-77. https://doi.org/10.1016/S0749-0720(02)00004-X.

Collie, D. D. 1992. Pulmonary function changes and clinical findings associated with chronic respiratory disease in calves. Br. Vet. J. 148:33-40. https://doi.org/10.1016/0007-1935(92)90064-8.

Dennis, M. J. 1986. The effects of temperature and humidity on some animal diseases-A review. Br. Vet. J. 142:472-485. https://doi .org/10.1016/0007-1935(86)90051-5.

Doyle, D., B. Credille, T. W. Lehenbauer, R. Berghaus, S. S. Aly, J. Champagne, P. Blanchard, B. Crossley, L. Berghaus, S. Cochran, and A. Woolums. 2017. Agreement among 4 sampling methods to identify respiratory pathogens in dairy calves with acute bovine respiratory disease. J. Vet. Intern. Med. 31:954-959. https://doi .org/10.1111/jvim.14683.

Eberhart, N. L., J. M. Storer, M. Caldwell, A. M. Saxton, and P. D. Krawczel. 2017. Behavioral and physiologic changes in Holstein steers experimentally infected with Mannheimia haemolytica. Am. J. Vet. Res. 78:1056-1064. https://doi.org/10.2460/ajvr.78.9.1056.

Gardner, B. A., H. G. Dolezal, L. K. Bryant, F. N. Owens, and R. A. Smith. 1999. Health of finishing steers: Effects on performance, carcass traits, and meat tenderness. J. Anim. Sci. 77:3168-3175.

Gershwin, L. J., M. L. Anderson, C. Wang, L. J. Berghaus, T. P. Kenny, and R. A. Gunther. 2011. Assessment of IgE response and cytokine gene expression in pulmonary efferent lymph collected after ovalbumin inhalation during experimental infection of calves with bovine respiratory syncytial virus. Am. J. Vet. Res. 72:134145. https://doi.org/10.2460/ajvr.72.1.134.

Gorden, P. J., and P. Plummer. 2010. Control, management, and prevention of bovine respiratory disease in dairy calves and cows. Vet. Clin. North Am. Food Anim. Pract. 26:243-259. https://doi.org/ 10.1016/j.cvfa.2010.03.004.

Lavallee, P., and J-F. Beaumont. 2015. Why We Should Put Some Weight on Weights. Survey Insights: Methods from the Field, Weighting: Practical Issues and 'How to' Approach, Invited article. Accessed Mar. 23, 2018. http://surveyinsights.org/?p=6255.

Lever, J. K. M., and N. Altman. 2016. Model selection and overfitting. Nat. Methods 13:703-704.

Love, W. J., T. W. Lehenbauer, P. H. Kass, A. L. Van Eenennaam, and S. S. Aly. 2014. Development of a novel clinical scoring system for on-farm diagnosis of bovine respiratory disease in pre-weaned dairy calves. PeerJ 2:e238. https://doi.org/10.7717/peerj.238.

Love, W. J., T. W. Lehenbauer, A. L. Van Eenennaam, C. M. Drake, P. H. Kass, T. B. Farver, and S. S. Aly. 2016. Sensitivity and specificity of on-farm scoring systems and nasal culture to detect bovine respiratory disease complex in preweaned dairy calves. J. Vet. Diagn. Invest. 28:119-128. https://doi.org/10.1177/ 1040638715626204

Masseau, I., G. Fecteau, L. Breton, P. Helie, G. Beauregard, and L. Blond. 2008. Radiographic detection of thoracic lesions in adult 
cows: A retrospective study of 42 cases (1995-2002). Can. Vet. J. 49:261-267.

McGuirk, S. M., and S. F. Peek. 2014. Timely diagnosis of dairy calf respiratory disease using a standardized scoring system. Anim. Health Res. Rev. 15:145-147. https://doi.org/10.1017/ S1466252314000267.

Moons, K. G., F. E. Harrell, and E. W. Steyerberg. 2002. Should scoring rules be based on odds ratios or regression coefficients? J. Clin. Epidemiol. 55:1054-1055. https://doi.org/10.1016/S0895 -4356(02)00453-5.

National Oceanic and Atmospheric Administration (NOAA). 2018. Local Climatological Data Publication. Accessed Mar. 23, 2018. http://www.noaa.gov/weather.

Ollivett, T. L., and S. Buczinski. 2016. On-farm use of ultrasonography for bovine respiratory disease. Vet. Clin. North Am. Food Anim. Pract. 32:19-35. https://doi.org/10.1016/j.cvfa.2015.09.001.

Ollivett, T. L., J. L. Caswell, D. V. Nydam, T. Duffield, K. E. Leslie, J. Hewson, and D. Kelton. 2015. Thoracic ultrasonography and bronchoalveolar lavage fluid analysis in Holstein calves with subclinical lung lesions. J. Vet. Intern. Med. 29:1728-1734. https://doi .org/10.1111/jvim. 13605

Panciera, R. J., and A. W. Confer. 2010. Pathogenesis and pathology of bovine pneumonia. Vet. Clin. North Am. Food Anim. Pract. 26:191-214. https://doi.org/10.1016/j.cvfa.2010.04.001.

Powers, D. M. W. 2007. Mach. Learn. Technol. Evaluation: From Precision, Recall and F-Factor to ROC, Informedness, Markedness \& Correlation. School of Informatics and Engineering, Flinders University, Adelaide, Australia.
Rabeling, B., J. Rehage, D. Döpfer, and H. Scholz. 1998. Ultrasonographic findings in calves with respiratory disease. Vet. Rec. 143:468-471.

Snowder, G. D., L. D. Van Vleck, L. V. Cundiff, and G. L. Bennett. 2006. Bovine respiratory disease in feedlot cattle: Environmental, genetic, and economic factors. J. Anim. Sci. 84:1999-2008. https:/ /doi.org/10.2527/jas.2006-046.

Thomas, L. H., E. J. Stott, A. P. Collins, N. J. Jebbett, and A. J. Stark. 1977. Evaluation of respiratory disease in calves: Comparison of disease response to different viruses. Res. Vet. Sci. 23:157164.

USDA. 2010. Dairy 2007, Heifer Calf Health and Management Practices on U.S. Dairy Operations, 2007 USDA:APHIS:VS, CEAH. Fort Collins, CO \#550.0110.

USDA. 2012. Dairy Heifer Raiser, 2011 USDA-APHIS-VS, CEAH, National Animal Health Monitoring System (NAHMS), Fort Collins, CO \#613.1012.

Verhoeff, J., A. Wierda, and A. P. van Nieuwstadt. 1985. Correlation of a disease scoring system with arterial $\mathrm{PO}_{2}$ values in respiratory syncytial virus infection in calves. Vet. Q. 7:106-111. https://doi .org/10.1080/01652176.1985.9693965.

Wittum, T. E., N. E. Woollen, L. J. Perino, and E. T. Littledike. 1996. Relationships among treatment for respiratory tract disease, pulmonary lesions evident at slaughter, and rate of weight gain in feedlot cattle. J. Am. Vet. Med. Assoc. 209:814-818. 